\title{
Absceso cerebral por Scedosporium apiospermum. Presentación de un caso con revisión de la literatura
}

M. Gelabert-González; J. Llovo-Taboada*; R. Reyes-Santías**; A. Arcos-Algaba; R. Serramito-García; M.D. Peñalver-Barral* y A. García-Allut

Servicios de Neurocirugía, Microbiología* y Patología**. Complejo Hospitalario Universitario de Santiago de Compostela. Universidad de Santiago de Compostela.

\section{Resumen}

Scedosporium apiospermum es un hongo que se encuentra en el suelo y en las aguas contaminadas, que habitualmente ocasiona infecciones cutáneas y excepcionalmente puede contaminar el sistema nervioso central. Sus infecciones se relacionan habitualmente con situaciones de inmunosupresión. Los autores presentan el caso de una mujer de $\mathbf{7 3}$ años con insuficiencia renal crónica que se presentó con dolor de cabeza durante 2 semanas y que en la tomografía computarizada se observó una lesión en anillo en el lóbulo temporal izquierdo. Con el diagnostico de absceso cerebral se practicó una craneotomía temporal y extirpación completa de la lesión. La paciente falleció 5 días después de la intervención. En el cultivo del material evacuado se aisló Scedosporium apiospermum. Se realiza una revisión de los casos publicados de absceso cerebral por Scedosporium apiospermum.

PALABRAS CLAVE: Absceso cerebral. Pseudallescheria boydii. Scedosporium apiospermum. Voriconazol.

Scedosporium apiospermum brain abscess. Report of one case with literature review

\section{Summary}

Scedosporium apiospermum is a fungus found in the soil and in contaminated water and commonly cause cutaneous infections and is a rare cause of central nervous system infection. Invasive infection is usually associated with immunosuppresion. The authors present a 73-year-old woman with chronic renal disease who presented with headache for 2 weeks. Computerized tomography scans revealed a ring-enhancing lesion in left temporal lobe. An urgent craniotomy was performed and the lesion was totally removed. The patient died 5 days later. Scedosporium apiospermum was isolated in

Recibido: 18-02-09. Aceptado: 14-04-09 the culture of the extirpated lesion.

The authors review the previously reported cases of brain abscess for Scedosporium apiospermum.

KEY WORDS: Brain abscess. Pseudallescheria boydii. Scedosporium apiospermum. Voriconazole.

Introducción

Los hongos que con mayor frecuencia causan infecciones del sistema nervioso central (SNC) son especies de Candida, Cryptococcus neoformans, Aspergillus fumigatus y Zigomicetos. Sin embargo, debido a la cada vez mayor presencia de pacientes inmunodeprimidos (sometidos a trasplante de órganos, afectados de SIDA, etc.), se han descrito otros hongos poco habituales como causantes directos de infecciones orgánicas ${ }^{25}$. Entre estos nuevos agentes Scedosporium apiospermum (SA) ocupa un lugar importante debido sobre todo a su extensión universal y a su capacidad para infectar huéspedes comprometidos.

El hongo Scedosporium apiospermum, conocido anteriormente como Monosporium apiospermum, Petriellidium boydii o Allescheria boydii ${ }^{24}$, es la forma asexual del Pseudallesceria boydii, un hongo saprofito, de distribución universal, frecuentemente aislado de los suelos, aguas estancadas y residuales ${ }^{8,2}$. Su infección más conocida es la forma subcutánea, aunque también puede ocasionar sinusitis, otitis, infecciones oculares o pulmonares, etc. tanto en pacientes inmunológicamente sanos como en situaciones de inmunosupresión ${ }^{1,6,23}$. El absceso cerebral por Scedosporium apiospermum es muy poco frecuente y en la revisión de la literatura sólo hemos encontrado 40 casos publicados desde 1953. Presentamos el caso de una mujer en insuficiencia renal crónica que presentó un absceso cerebral por SA y revisamos los casos notificados con anterioridad.

\section{Caso clínico}

Mujer de 73 años de edad con insuficiencia renal crónica secundaria a nefroesclerosis y sometida a hemodiálisis desde hace 4 años. Acude a urgencias del hospital por 


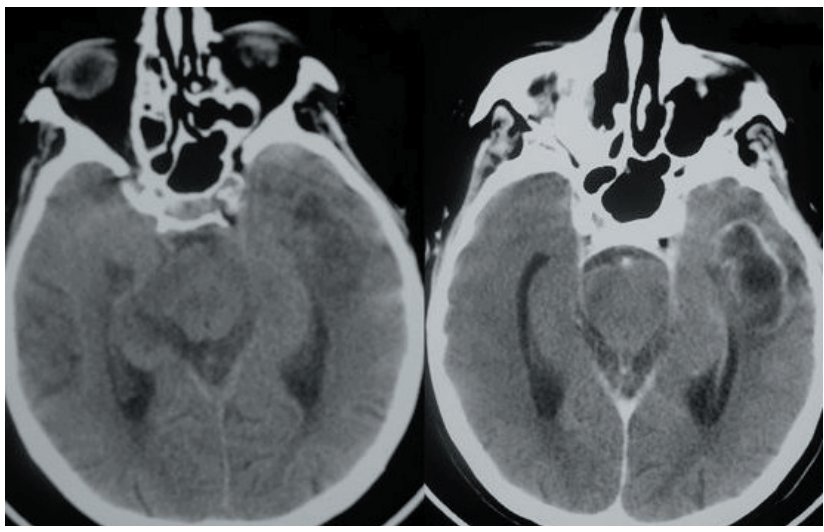

Figura 1. Tomografia computarizada sin y con contraste mostrando el absceso en lóbulo temporal izquierdo.

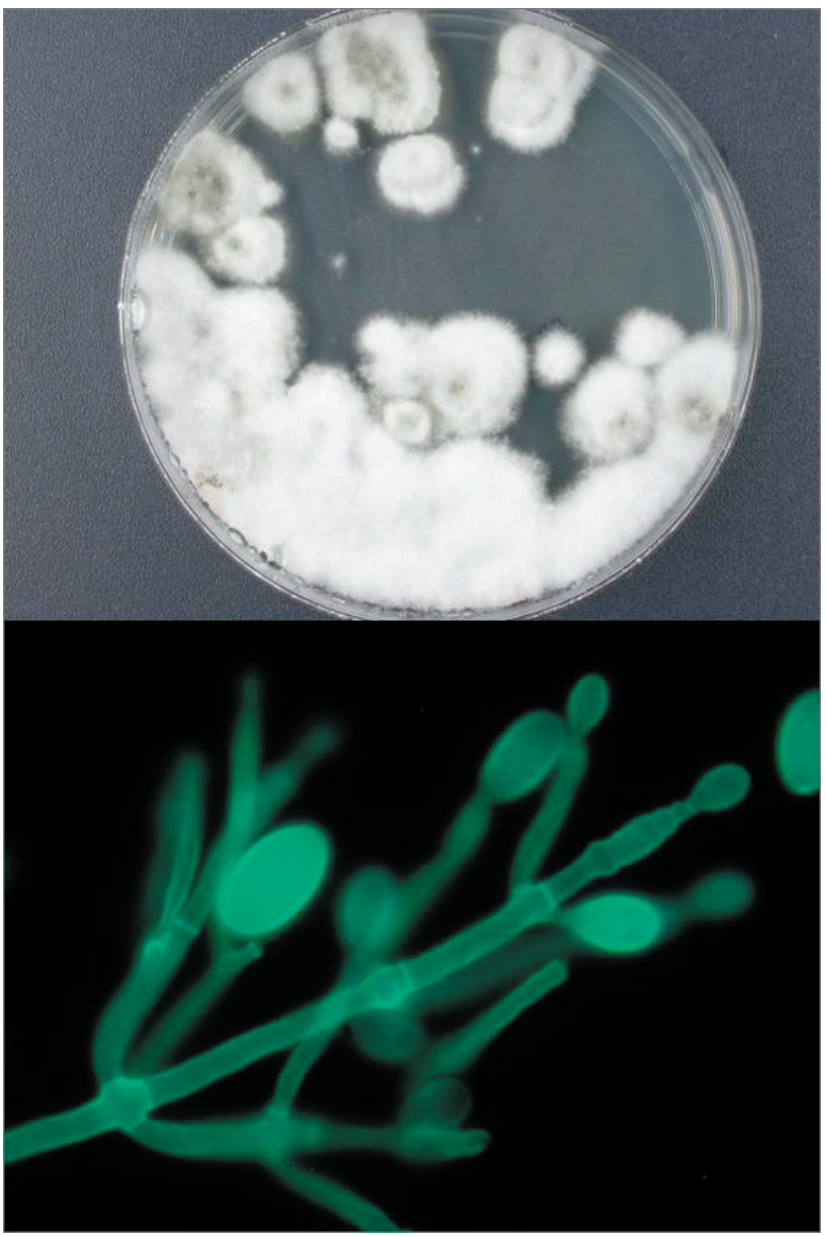

Figura 2. Superior: cultivo de SA en medio de Sabouraud. Inferior: micelios, células conidiogenas y conidios teñidos con calcofluor White (1000x)

presentar dolor de cabeza durante los últimos 2 días. El examen neurológico al ingreso fue normal. Se practicó un estudio con tomografía computarizada craneal que reveló una lesión hipodensa que se realzaba de forma irregular con

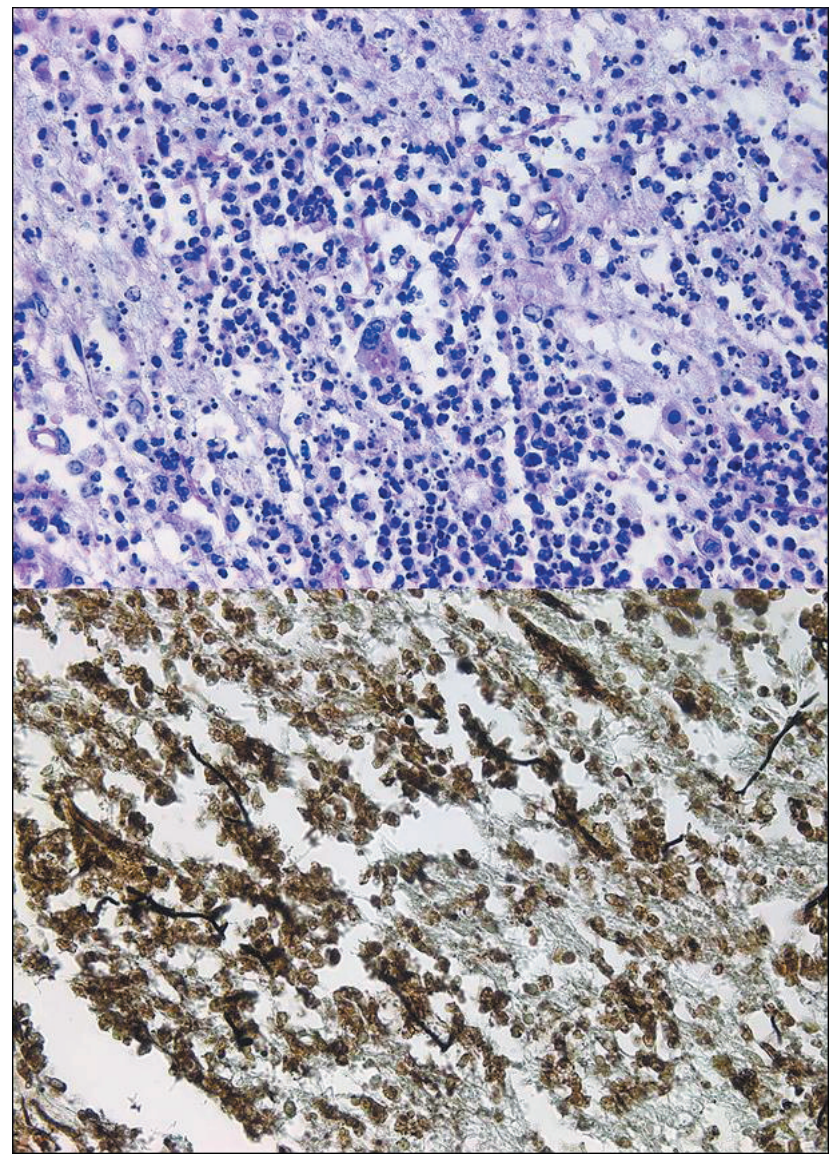

Figura 3. Microfotografia con tinciones de PAS (superior) y Grocott (inferior) mostrando la presencia de hifas.

contraste y rodeada de edema vasogénico (Figura 1). Con el diagnóstico de absceso cerebral se realizó una craneotomía temporal y exéresis completa de una lesión quística con contenido purulento y sugestiva de absceso. Se inició tratamiento con vancomicina, ceftazidima y metronidazol de forma inmediata.

El postoperatorio inicial fue satisfactorio, la paciente se extubó a las 24 horas sin objetivarse déficits. A las 48 horas de la cirugía presenta una crisis motora generalizada e importante disminución en el nivel de conciencia. Una nueva tomografía axial computarizada mostró una hemorragia en la cavidad operatoria, por lo que la paciente fue llevada nuevamente a quirófano para evacuación del hematoma. La paciente no recuperó la conciencia y falleció a los 3 días. El estudio histológico del material extirpado mostró cambios reactivos y linfohistiocitarios con infiltrado perivascular y células inflamatorias. El estudio microbiológico mostró un Scedosporium apiospermum sensible al voriconazol (Figura 2).

El estudio necrópsico de la paciente evidenció hemorragias en el lóbulo temporal izquierdo, intraventricular y subaracnoidea. Microscópicamente sobre el lóbulo tempo- 
ral izquierdo existía un infiltrado inflamatorio de predominio linfoide y de localización perivascular. Existían focos con reacción gigantocelular asociada. Las técnicas de PAS y Grocott ponen de manifiesto la presencia de hifas septadas y ramificadas (Figura 3).

\section{Discusión}

En las dos últimas décadas, se ha producido un marcado aumento en el número de infecciones fúngicas del SNC y a la participación de nuevas especies de hongos, debido al creciente número de pacientes inmunodeprimidos ${ }^{25,6}$. La primera infección por SA en el sistema nervioso fue descrita en 1953 por Aronson et al. ${ }^{4}$, en un paciente de 46 años que se presentó con meningitis crónica después de ser sometido a una anestesia epidural. Aunque Winston et al. ${ }^{41}$ diagnosticaron por autopsia un absceso por Pseudallescheria boydii, el primer caso diagnosticado en vida fue publicado en 1978 por Bell y Myers ${ }^{6}$ en un niño de 3 años con leucemia linfocítica aguda. Con inclusión de la paciente descrita en este artículo, únicamente se han descrito 41 casos de absceso cerebral por SA.

De los 40 casos revisados, 27 eran varones y 12 mujeres (en una referencia no se incluye el sexo), con edades comprendidas entre 1.5 y 69 años (media de 28); 17 presen-

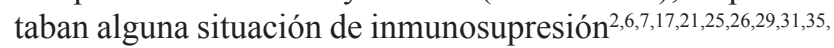
$36,39,41,42,8$ por haberse sometido a un trasplante $2,19,23,24,28,34,38$, 7 que sufrían un linfoma o leucemia ${ }^{6,7,26,34,36,41,42}$, y los otros dos pacientes correspondían a un caso de cirrosis hepática ${ }^{17}$ y a un tratamiento prolongado con corticosteroides ${ }^{31}$. En los otros 23 pacientes no había antecedentes de enferme$\operatorname{dad}^{1,3,5,8,10-13,15,16,22,23,27,28,33,38,40}$, en estos casos el ahogamiento fue el principal factor predisponente en 19 pacientes $^{5,8,10-}$ 13,15,19,21-23,27,28,33,38,40, seguidos de 2 casos de lesión penetrante ocular $^{3,13}$. Las infecciones del SNC por Pseudallesceria boydii pueden ocasionar abscesos cerebrales ${ }^{10}$, meningitis ${ }^{22}$, ventriculitis / ependimitis ${ }^{28}$, habiéndose descrito un caso de contaminación de una derivación ventrículo-peritoneal ${ }^{32}$. Dentro del sistema nervioso los abscesos pueden localizarse en hemisferios cerebrales ${ }^{26}$, el cerebelo ${ }^{13,41}$, el tronco cerebral $^{11} \mathrm{o}$ en la medula espinal ${ }^{14} \mathrm{y}$ por lo general son clínicamente expresivos, presentando alteraciones en el nivel de conciencia, signos de irritación meníngea y deficiencias neurológicas focales?

La fuente de diseminación al SNC es poco conocida en las especias de Scedosporium ya que los cultivos en sangre son habitualmente negativos. El Scedosporium prolificans sí libera conidios en sangre, de ahí su facilidad en producir infecciones diseminadas. La puerta pulmonar de diseminación se asume en la mayoría de los casos, aunque ha sido muy pocas veces demostrado. Los abscesos pueden ser múltiples como ocurrió en 26 de los casos revisados ${ }^{1-3,5,8,10,12,13,16,17,19,22-27,33,35,38,40-42}$ o único (14 casos $)^{2,6,7,11,12,14,20,27,28,30,33,35,38}$.

Las opciones de tratamiento son similares a las adoptadas para todos los abscesos cerebrales independientemente del germen casual e incluyen cirugía, ya sea escisión o aspiración, seguidas de la terapia antifúngica adecuada ${ }^{18}$. De los 40 casos publicados, 13 casos fueron tratados exclusivamente con terapia antifúngica ${ }^{2,6,11,16,17,22,24,25,35,39-41}$, 22 sometidos a cirugía seguida de terapia especifica ${ }^{1,3,5-}$ $7,10,12,13,19,21,23,26-29,31,35$, dos pacientes no recibieron tratamiento especifico siendo diagnosticados en autopsia ${ }^{36,42} \mathrm{y}$ dos casos que no especifica el tratamiento ${ }^{20,33}$. Entre los pacientes intervenidos en 19 de ellos se realizó resección más o menos amplia ${ }^{3,6,7,10,12,13,19,21-23,26-29,35}, 3$ casos con punción-aspiración ${ }^{1,5,8}$ siendo 2 con técnica estereotáxica ${ }^{5,8}$.

En la literatura existe controversia sobre cual es el tratamiento idóneo para la infección por Pseudallesceria boydii. El organismo es generalmente resistente a la anfotericina $\mathrm{B}$, fluconazol y flucitosina, aunque existen algunos datos sobre una buena respuesta a la anfotericina ${ }^{2,11}$. El Itraconazol tiene una actividad variable pero de todas formas las concentraciones que se alcanzan en el líquido cefalorraquídeo son menores del $1 \%$ de las concentraciones séricas.

El miconazol tiene también una pobre penetración meníngea por lo que su indicación es emplearlo directamente por vía intratecal, usando una dosis recomendada para adultos de $20 \mathrm{mg}$, detectándose niveles por encima de la concentración mínima inhibidora en el líquido cefalorraquídeo a las 48 horas después de su administración por esta vía ${ }^{27}$. Recientemente, el voriconazol ha surgido como una posible opción de tratamiento en casos de pseudallescheriasis del sistema nervioso central debido a su buena difusión a través de la barrera hematoencefálica en pacientes con meningitis fúngica, su actividad in vitro es más potente que la del fluconazol contra una alta variedad de hongos ${ }^{25}$, sin embargo tiene un mayor potencial de efectos tóxicos, sobre todo a nivel hepático, observándose un incremento dosis-dependiente en el nivel de la fosfatasa alcalina hasta en el $20 \%$ de los pacientes ${ }^{29,30}$. Su metabolismo es a través del enzima del citocromo $\mathrm{P} 450$ por lo que presenta interacciones con algunos fármacos empleados en pacientes transplantados como la ciclosporina o el tacrolimus, por lo que suele ser necesario ajustes estrictos de dosis $^{30}$. El posaconazol es otro azol de segunda generación que ha demostrado ser activo contra Pseudallescheria boydii y aunque hay pocas referencias sobre su penetración en el sistema nervioso, se ha utilizado con éxito para el tratamiento de abscesos cerebrales por este hongo ${ }^{26}$. La actividad de la caspofungina, encuadrada dentro del grupo de las echinocandinas, contra Pseudallescheria no esta perfectamente aclarada ${ }^{28}$, sin embargo se ha recomendado la combinación de caspofungina y variconazo ${ }^{34} \mathrm{y}$ aún no se ha publicado su empleo intratecal, por el contrario sí se ha 


\begin{tabular}{|c|c|c|c|c|c|c|c|c|c|c|c|c|c|c|c|c|c|c|c|c|c|c|}
\hline \multirow{8}{*}{ 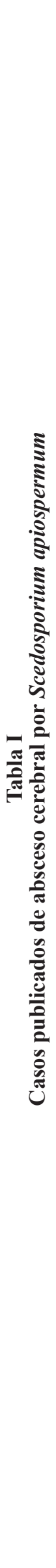 } & 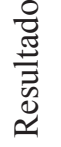 & 芠 &.$\overline{\frac{D}{n}}$ & 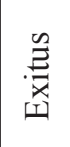 & $\begin{array}{l}\text { 芠 } \\
\text { 离 }\end{array}$ & 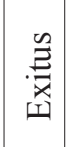 &.$\overline{\frac{D}{\omega}}$ & $\begin{array}{l}\text { 䒝 } \\
\text { | }\end{array}$ & $\begin{array}{l}\text { 足 } \\
\text { 离 }\end{array}$ & 芠 & $\begin{array}{l}\text { 芑 } \\
\text { 离 }\end{array}$ & 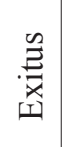 & $\begin{array}{l}\text { 离 } \\
\text { 齐 }\end{array}$ & 节 & 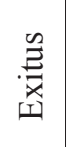 & 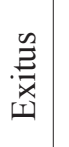 & 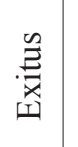 &. $\bar{\Xi}$ &. $\bar{\Xi}$ & 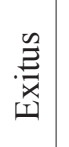 & 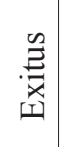 & $\begin{array}{l}\text { 芠 } \\
\text { 齐 }\end{array}$ \\
\hline & 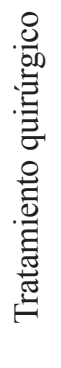 & 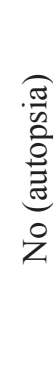 & 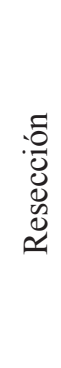 & $\grave{z}$ & z & $z$ & 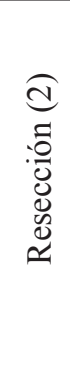 & 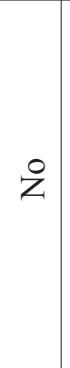 & 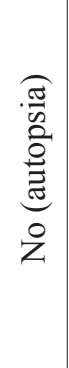 & $\stackrel{z}{z}$ & 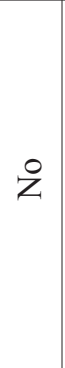 & 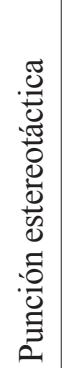 & 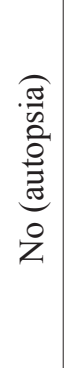 & $\ddot{i n}$ & 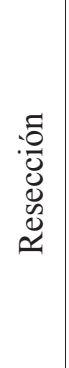 & 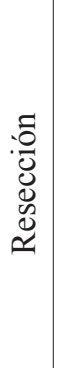 & 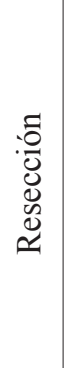 & 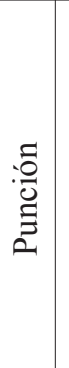 & 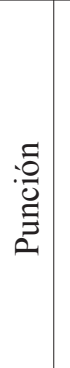 & 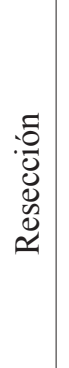 & 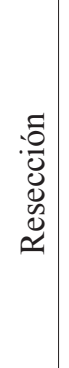 & 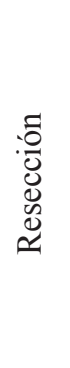 \\
\hline & 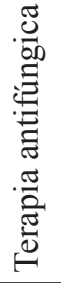 & $\stackrel{8}{z}$ & 毒 & 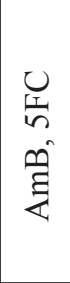 & 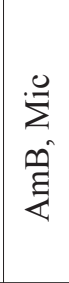 & $\overbrace{2}^{0}$ & $\begin{array}{l}\sum_{0}^{0} \\
\text { 罢 }\end{array}$ & 妾 & $\stackrel{\circ}{z}$ & 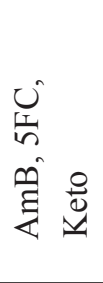 & $\stackrel{\mathscr{E}}{\Sigma}$ & 妾 & $\stackrel{0}{z}$ & $\stackrel{\mathscr{g}}{\Sigma}$ & 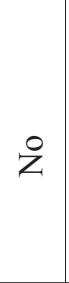 & $\dot{g}_{\Sigma}^{0}$ & 妾 & 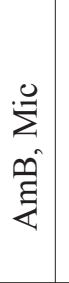 & $\stackrel{0}{\Sigma}$ & 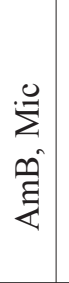 & 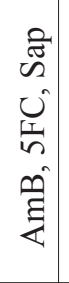 & 妾 \\
\hline & 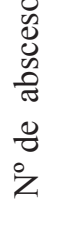 & $\begin{array}{l}\frac{0}{0} \\
\stackrel{\circ}{\circ}\end{array}$ & 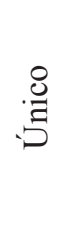 & : $\frac{0}{\tilde{n}}$ & 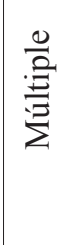 & $\begin{array}{l}0 \\
.0 \\
.5 \\
.5\end{array}$ & $\begin{array}{l}\frac{0}{0} \\
\frac{0}{0}\end{array}$ & 竞 & 惫 & 莺 & 旁 & 善 & $\begin{array}{l}.0 \\
.0 \\
.5\end{array}$ & 范 & 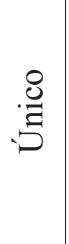 & . & 总 & 总 & 竞 & 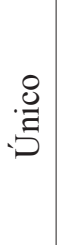 & 总 & 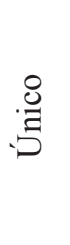 \\
\hline & 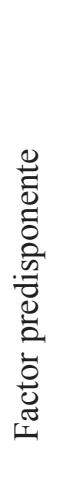 & 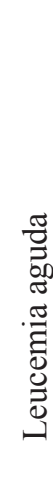 & 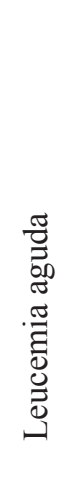 & 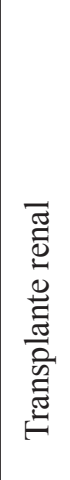 & $\begin{array}{l}0 \\
.0 \\
0 \\
0 \\
0 \\
0 \\
0 \\
0 \\
0\end{array}$ & 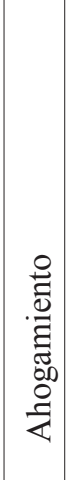 & 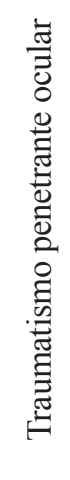 & 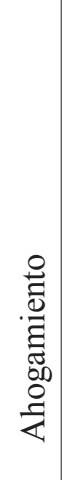 & 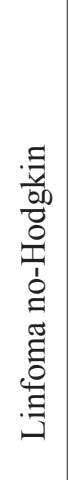 & 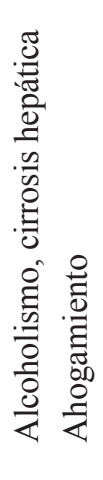 & 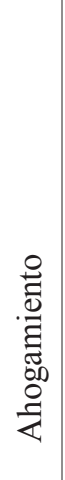 & 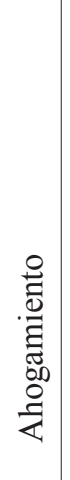 & 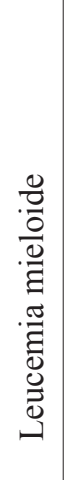 & 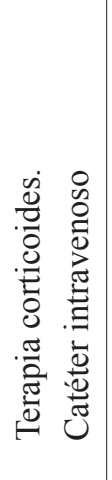 & 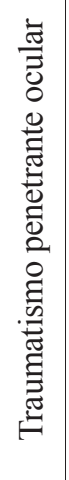 & 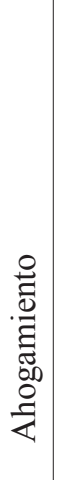 & 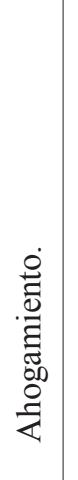 & 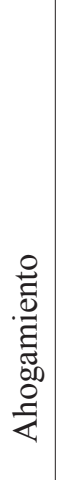 & 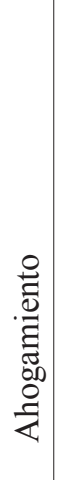 & 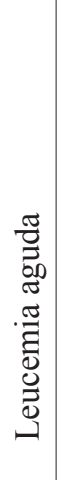 & 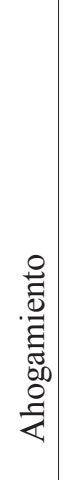 & 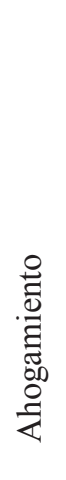 \\
\hline & 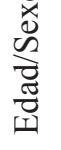 & $\underset{i n}{i}$ & $z_{m}$ & $\underset{m}{\gtrless}$ & $\sum_{\delta}$ & $\underset{\Omega}{z}$ & $\gtrless$ & ત્ત & 唕 & 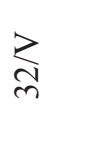 & $\begin{array}{l}3 \\
\text { o }\end{array}$ & 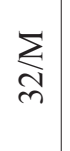 & $\underset{⿱ 乛}{\gtrless}$ & 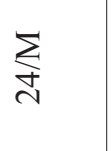 & $\underset{\sim}{\mathrm{n}}$ & 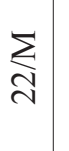 & 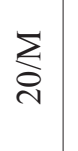 & ২্৯ & సે & $\underset{\substack{\infty \\
\sim}}{\sum_{j}}$ & $\underset{\exists}{\sum_{J}}$ & 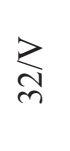 \\
\hline & 宗 & 5 & $\stackrel{\infty}{\stackrel{2}{a}}$ & $\begin{array}{l}\infty \\
\stackrel{\infty}{2}\end{array}$ & $\vec{\infty}$ & $\stackrel{\mathscr{D}}{\stackrel{\Omega}{\sigma}}$ & $\begin{array}{l}+ \\
\stackrel{D}{\sigma}\end{array}$ & 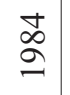 & $\begin{array}{l}\infty \\
2\end{array}$ & $\stackrel{\infty}{2}$ & $\begin{array}{l}\mathscr{2} \\
\stackrel{2}{a}\end{array}$ & $\begin{array}{l}\mathscr{2} \\
2 \\
\varrho\end{array}$ & $\stackrel{\circ}{\stackrel{\sigma}{\sigma}}$ & $\stackrel{\infty}{\stackrel{一}{\sigma}}$ & $\begin{array}{l}\infty \\
\stackrel{\infty}{\varrho}\end{array}$ & $\stackrel{\partial}{\stackrel{\partial}{a}}$ & $\stackrel{\circ}{\circ}$ & $\begin{array}{l}\stackrel{\partial}{\sigma} \\
\stackrel{\sigma}{2}\end{array}$ & $\begin{array}{l}\stackrel{\alpha}{\sigma} \\
\stackrel{\sigma}{\sigma}\end{array}$ & $\begin{array}{l}\stackrel{a}{\sigma} \\
\stackrel{\sigma}{\sigma}\end{array}$ & $\stackrel{2}{\circ}$ & $\stackrel{\circ}{\circ}$ \\
\hline & 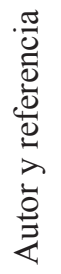 & 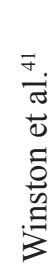 & 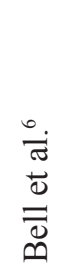 & 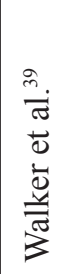 & 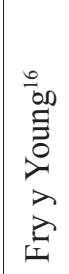 & 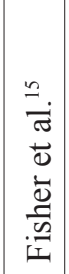 & 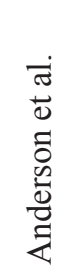 & 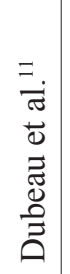 & 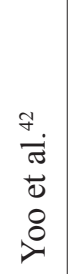 & $\begin{array}{l}\overrightarrow{\bar{\pi}} \\
\overline{0} \\
\overrightarrow{\tilde{E}} \\
\tilde{0}\end{array}$ & 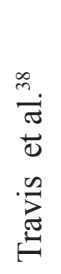 & 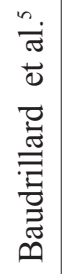 & 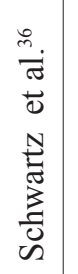 & $\begin{array}{l}\overrightarrow{0} \\
\vec{\sigma} \\
\overrightarrow{0} \\
\overrightarrow{0} \\
\overrightarrow{0} \\
\overrightarrow{0}\end{array}$ & 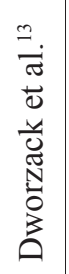 & 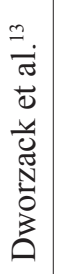 & 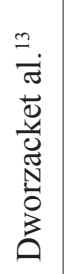 & 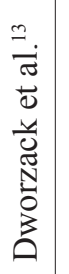 & 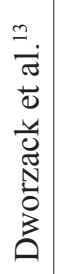 & 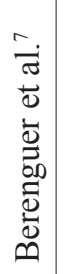 & 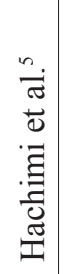 & 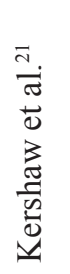 \\
\hline
\end{tabular}




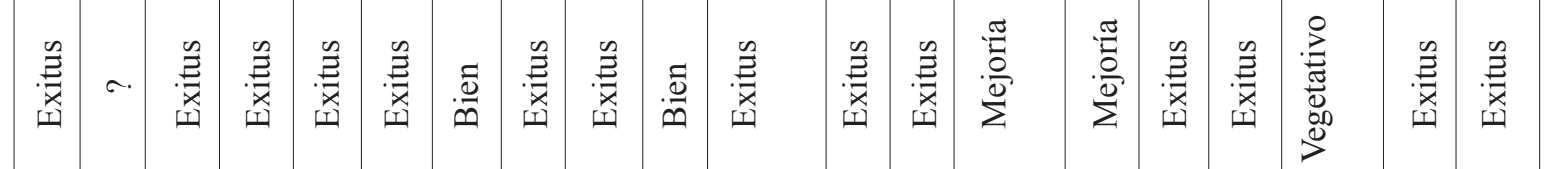
L. 总

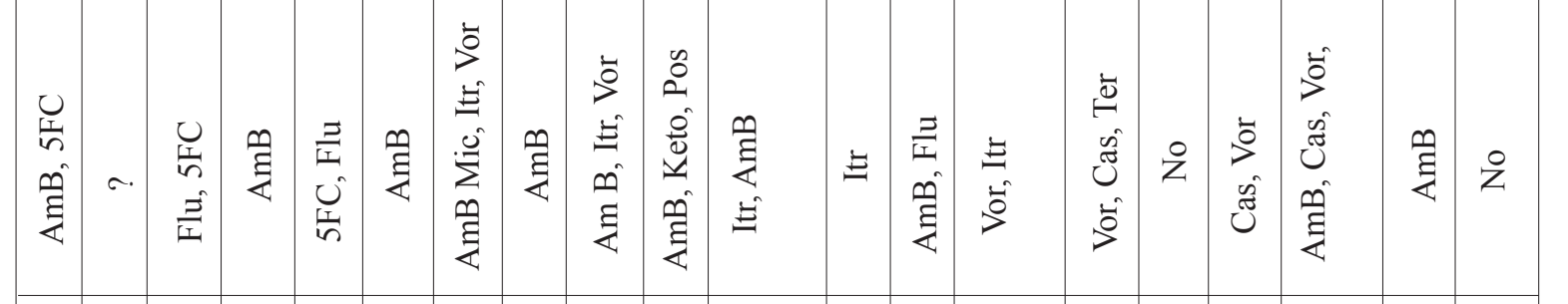

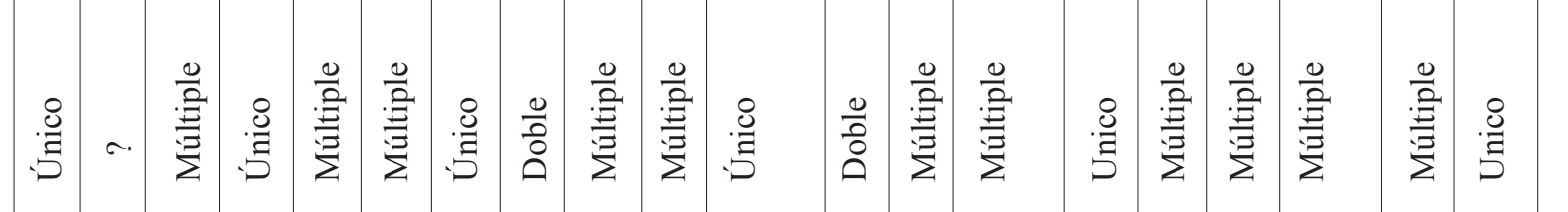
这 离 $\ddot{E}$

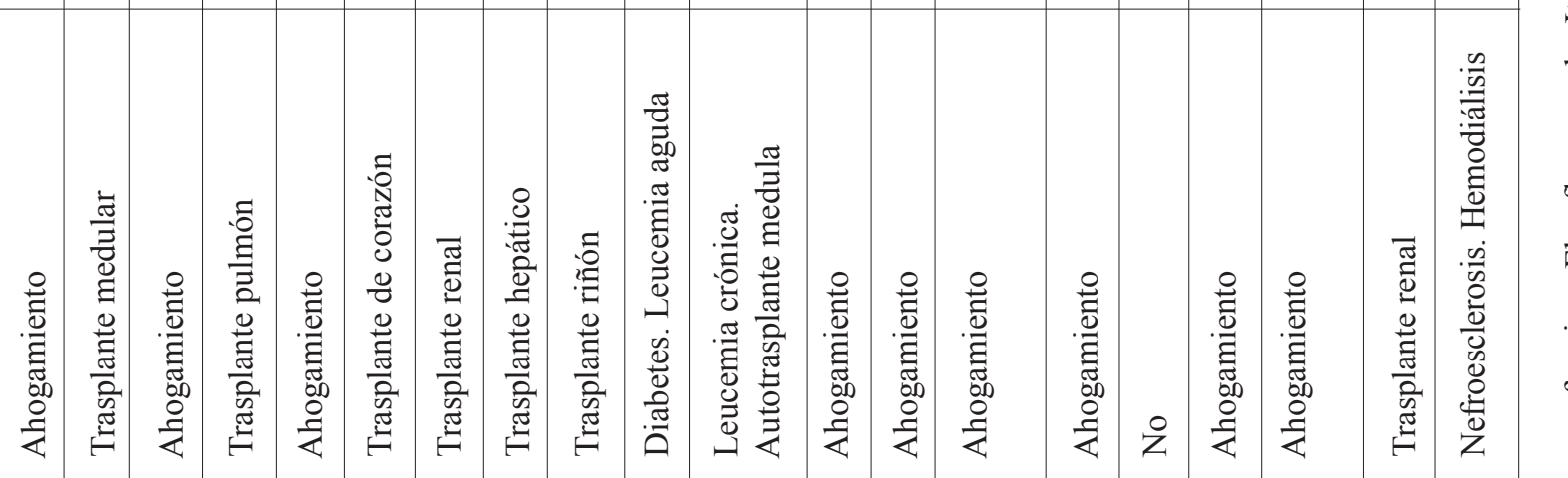
is

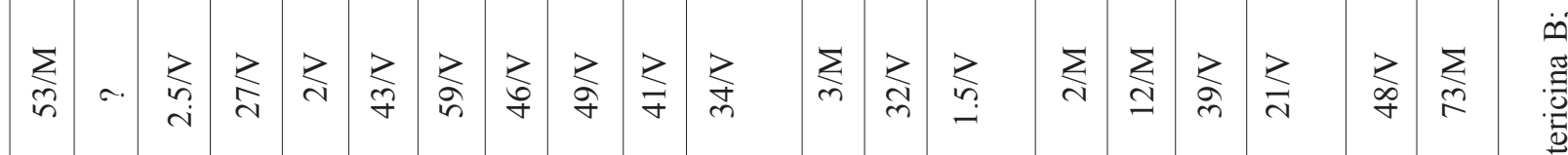

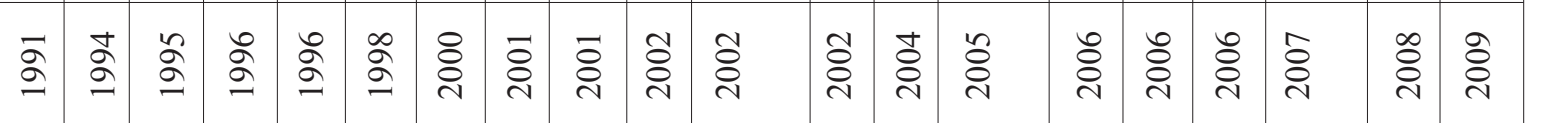

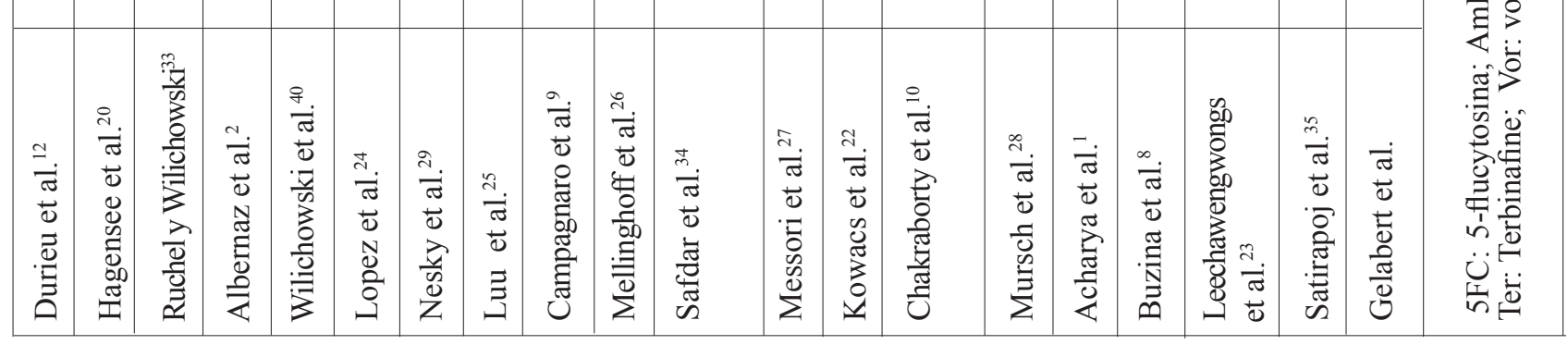


utilizado el miconazol inratecal con buen resultado en dos casos de absceso cerebral por Pseudallescheria boydii ${ }^{13,31}$. Otro aspecto controvertido es la duración del tratamiento aunque en la mayoría de los casos se aconseja un mínimo de 2 meses $^{29}$. El pronóstico es en general bastante malo, de los 40 casos analizados, 30 fallecieron (72.5\%), 9 sobrevivieron aunque en un caso en situación vegetativa ${ }^{22} \mathrm{y}$ en el paciente restante no se especifica el resultado ${ }^{20}$.

\section{Conclusiones}

La infección del sistema nervioso por Scedosporium apiospermum es un cuadro clínico poco frecuente y de extrema gravedad. Su presencia debe sospecharse cuando aparece un absceso cerebral en un paciente inmunocomprometido y que no responde de forma adecuada a la terapéutica antibiótica habitual. A pesar del empleo de un tratamiento agresivo de cirugía y terapia antifúngica la mortalidad es muy elevada.

\section{Bibliografía}

1. Acharya, A., Ghimire, A., Khanal, B., Bhattacharya, S., Kumari, N., Kanun, R.: Brain abscess due to Scedosporium apiospermum in a non immunocompromised child. J Med Microbiol 2006; 24: 231-236.

2. Albernaz, V., Huston, B., Castillo, M., Mukherjim S., Bouldin, T.W.: Pseudallescheria boydii infection of the brain: imaging with pathologic confirmation. Am J Neuroradiol 1996; 17: 589-592.

3. Anderson, R.L., Carroll, T.F., Harvey, J.T., Myers, M.G.: Petriellidium (Allescheria) boydii orbital and brain abscess treated with intravenous miconazole. Am J Ophthalmol 1984; 97: 771-775.

4. Aronson, S.M., Benham, R., Wolf, A.: Maduromycosis of the central nervous system. J Neuropathol Exp Neurol 1953; 12: 158-168.

5. Baudrillard, J.C., Rousseaux P., Lerais JM et al: Anéurysmes mycotiques fongiques et abcès cérébraux multiples à Scedosporium Apiospermum. A propos d'une observation avec revue de la literature. J Radiol 1985; 66: 321-326.

6. Bell, W.E., Myers, M.G.: Allescheria (Petriellidium) boydii brain abscess in a child with leukemia. Arch Neurol 1978; 35: 386-388.

7. Berenguer, J., Diaz-Mediavilla, J., Urra, D., Muñoz, P.: Central nervous system infection caused by Pseudallescheria boydii: case report and review. Rev Infect Dis 1989; 11: 890896.

8. Buzina, W., Feierl, G., Haas, D. et al.: Lethal brain abscess due to the fungus Scedosporium apiospermum (teleomorph Pseudallescheria boydii) after a near-drowning incident: case report and review of the literature. Med Mycol 2006; 44: 473-477.
9. Campagnaro, E.L., Woodside, K.J., Early et al.: Disseminated Pseudoallescheria boydii (Scedosporium apiospermum) infection in a renal transplant patient. Transpl Infect Dis 2002; 4: 207-211.

10. Chakraborty, A., Worman, M.R., Bullock, P,R.: Scedosporium apiospermum brain abscess treated with surgery and voriconazole. J Neurosurg (Pediatrics) 2005; 103: 83-87

11. Dubeau, F., Roy, L.E., Allard, J., et al.: Brain abscess due to Petriellidium boydii. Can J Neurol Sci 1984; 11: 395398.

12. Durieu, I., Parent, M., Ajana, F., et al.: Monosporium apiospermum meningoencephalitis: a clinic-pathological case. J Neurol Neurosurg Psychiatry 1991; 54: 731-733.

13. Dworzack, D.L., Clark, B.B., Borkowski, W,J. et al.: Pseudallescheria boydii brain abscess: association with neardrowing and efficacy of high dose, prolonged miconazole therapy in patients with multiple abscesses. Medicine (Baltimore) 1989; 68: 218-224.

14. Fietz, T., Knauf, W., Schwartz, S., Thiel, E.: Intramedullary abscess in a patient with disseminated Scedosporium apiospermum infection. Br J Haematology 2003; 120: 724.

15. Fisher, J.F., Shadomy, S., Teabeaut, J.R., et al.: Neardrowning complicated by brain abscess due to Petriellidium boydii. Arch Neurol 1982; 39: 511-513.

16. Fry, V.G., Young, C..: A rare fungal brain abscess in an uncompromised host. Surg Neurol 1981; 15: 446-449.

17. Gari, M., Fruit, J., Rousseaux, P., et al.: Scedosporium (Monosporium) apiospermum: multiple brain abscesses. Sabouraudia 1985; 23: 371-376.

18. González, J.. Gelabert, M., Pravos, A.G., Villa, J.F.: Colecciones purulentas intracraneales. Revisión de 100 casos. Rev Neurol 1999; 29: 416-424.

19. Hachimi-Idrissi, S., Willemsen, M., Desprechins, B., et al.: Pseudallescheria boydii and brain abscesses. Pediatr Infect Dis J 1990; 9: 737-741.

20. Hagensee, M.E., Bauwens, J.E., Kjos, B., Bowden, R.A.: Brain abscess following marrow transplantation: Experience at the Fred Hutchinson Cancer Research Center, 19841992. Clin Infect Dis 1994; 19: 402-408.

21. Kershaw, P., Freeman, R., Templeton, D., et al: Pseudallescheria boydii infection of the central nervous system. Arch Neurol 1990; 47: 468-472.

22. Kowacs, P.A., Soares, C.E., Monteiro de Almeida, S. et al.: Infection of the CNS by Scedosporium apiospermum after near drowning. Report of a fatal case and analysis of its cofounding factors. J Clin Pathol 2004; 57: 205-207.

23. Leechawengwongs, M., Milindankura, S., Liengudom, A., Chanakul, K., Viranuvatti, K., Clongsusuek, P.: Multiple Scedosporium apiospermum brain abscesses after near-drowning successfully treated with surgery and long-term voriconazole: a case report. Med Mycol 2006; 44: 295-327.

24. Lopez, F.A., Crowley, R.S., Wastila, L., et al.: Scedosporium apiospermum (Pseudallescheria boydii) infection in a 
heart transplant recipient: a case of mistaken identity. J Heart Lung Transpl 1998; 17: 321-324.

25. Luu, K.K., Scott, I.U., Miller, D., Davis, J.L.: Endogenous Pseudallescheria boydii endophtalmitis in a patient with ring-enhancing brain lesions. Ophthalmic Surg Lasers 2001; 32: $325-329$.

26. Mellinghoff, I.K., Winston, D.J., Mukwaya, G., Schiller, G.J.: Treatment of Scedosporium apiospermum brain abscess with posaconazole. Clin Infct Dis 2002; 34: 1648-1650.

27. Messori, A., Lanza, C., De Nicola, M., et al.: Mycotic aneurysms as letal complication of brain pseudallescheriasis in a near-drowned child: a CT demonstration. Am J Neuroradiol 2002; 23: 1697-1699.

28. Mursch, K., Trnovec, S., Ratz, H. et al: Successful treatment of multiple Pseudallescheria boydii brain abscesses and ventriculitis/ependymitis in a 2-year-old child after a neardrowing episode. Childs Nerv Syst 2006; 22: 189-192.

29. Nesky, M.A., McDougal, E.C., Peacock, J.E. Jr.: Pseudallescheria boydii brain abscess successfully treated with voriconazole and surgical drainage: case report and literature review of central nervous system pseudallescheriasis. Clin Infect Dis 2000; 31: 673-677.

30. O'Bryan, T.A.: Pseudallescheriasis in the 21 st century. Expert Rev Anti Infect Ther 2005; 3: 765-773.

31. Perez, R.E., Smith, M., McClendon, J., Kim, J., Eugenio, N.: Pseudallescheria boydii brain abscess: complication of an intravenous catheter. Am J Med 1988; 84: 359-362.

32. Peters, B., Faggett, T., Sampson, C.C.: Isolation of Monosporium apiospermum from cerebrospinal fluid. J Natl Med Assoc 1976; 68: 512-513.

33. Rüchel, R., Wilichowski, E.: Cerebral Pseudoallescheria mycosis after near-drowning. Mycoses 1995; 38: 473-478.

34. Safdar, A., Papadopoulos, E.B., Young, J.W.: Breakthrough Scedosporium apiospermum (Pseudallescheria boydii) brain abscess during therapy for invasive pulmonary aspergilosis following high-risk allogenic hematopoietic stem cell transplantation. Scedosporiasis and recent advances in antifungal therapy. Transpl Infect Dis 2002; 4: 212-217.

35. Satirapoj, B., Ruangkanchanasetr, P., Treewatcharee- korn, S., Supasyndh, O., Luesutthiviboon, L., Supaporn, T.: Pseudallescheria boydii brain abscess in a renal transplant recipient: First case report in southeast Asia. Transp Proceed 2008; 40: 2425-2427.

36. Schwartz, D.A., Amenta, P.S., Finkelstein, S,D.: Cerebral Pseudallescheria boydii infection: unique occurrence fungus ball formation in the brain. Clin Neurol Neurosurg 1989; 91: 79-84.

37. Selby, R.: Pachymeningitis secondary to Allescheria boydii. Case report. J Neurosurg 1972; 36: 225-227.

38. Travis, L.B., Roberts, G.D., Wilson, W.R.: Clinical significance of Pseudallescheria boydii: a review of 10 years ' experience. Mayo Clin Proc 1985; 60: 531-537.

39. Walker, D.H., Adamec, T., Krigman, M.: Disseminated petriellidosis (allescheriosis). Arch Pathol Lab Med 1978; 102: $158-160$.

40. Wilichowski, E., Hans-Jurgen, C., Schiffmann, H., Schulz-Schaeffer, W., Behrens-Baumann, W.: Fatal Pseudallescheria boydii panencephalitis in a child after near-drowning. Ped Infect Dis J 1996; 15: 365-370.

41. Winston, D.J., Jordan, M.C., Rhodes, J.: Allescheria boydii infections in the immunosuppressed host. Am J Med 1977; 63: 830-835.

42. Yoo, D., Lee, W.H., Kwon-Chung, K.J. Brain abscesses due to Pseudallescheria boydii associated with primary non-Hodgkin's lymphoma of the central nervous system a case report and literature review. Rev Infect Dis 1985; 7 : 272-277.

Gelabert-González, M.; Llovo-Taboada, J.; Reyes-Santías, R.; Arcos-Algaba, A.; Serramito-García, R.; Peñalver-Barral, M.D.; García-Allut, A.: Absceso cerebral por Scedosporium apiospermum. Presentación de un caso con revisión de la literatura. Neurocirugía 2010; 21: 125-131.

Correspondencia: Miguel Gelabert González. Servicio de Neurocirugía. Complejo Hospitalario de Santiago. La Choupana. 15706 Santiago de Compostela

miguel.gelabert@usc.es 